\title{
On vector potential of the Coulomb gauge
}

\author{
Valery P Dmitriyev \\ Lomonosov University, P.O. Box 160, Moscow 117574, Russid*
}

\begin{abstract}
The question of an instantaneous action (A M Stewart 2003 Eur. J. Phys. 24 519) can be approached in a systematic way applying the Helmhotz vector decomposition theorem to a two-parameter Lorenz-like gauge. We thus show that only the scalar potential may act instantaneously.
\end{abstract}

\section{INTRODUCTION}

The role of the gauge condition in classical electrodynamics was recently highlighted [1]. This is because of probable asymmetry between different gauges. The distinct feature of the Coulomb gauge is that it implies an instantaneous action of the scalar potential [2, 3, 4]. The question of simultaneous co-existence of instantaneous and retarded interactions is mostly debated [5]. The paper [2] concludes that 'the vector decomposition theorem of Helmholtz leads to a form of the vector potential of the Coulomb gauge that, like the scalar potential, is instantaneous'. This conclusion was arrived at considering the retarded integrals for electrodynamic potentials. Constructing within the same theorem wave equations the author 3] finds that 'the scalar potential propagates at infinite speed while the vector potential propagates at speed $c$ in free space'. In order to resolve the discrepancy between [2] and [3] the latter technique will be developed below in a more systematic way.

Recently the two-parameter generalization of the Lorenz gauge was considered [1, 4]:

$$
\boldsymbol{\nabla} \cdot \boldsymbol{A}+\frac{c}{c_{\mathrm{g}}^{2}} \frac{\partial \varphi}{\partial t}=0
$$

where $c_{\mathrm{g}}$ is a constant that may differ from $c$. We will construct wave equations applying the vector decomposition theorem to Maxwell's equations with (11). Thus simultaneous co-existence of instantaneous and retarded actions will be substantiated.

*Electronic address: dmitr@cc.nifhi.ac.ru 


\section{MAXWELL'S EQUATIONS IN THE KELVIN-HELMHOLTZ REPRESENTATION}

Maxwell's equations in terms of electromagnetic potentials $\mathbf{A}$ and $\varphi$ read as

$$
\begin{array}{r}
\frac{1}{c} \frac{\partial \boldsymbol{A}}{\partial t}+\boldsymbol{E}+\boldsymbol{\nabla} \varphi=0 \\
\frac{\partial \boldsymbol{E}}{\partial t}-c \boldsymbol{\nabla} \times(\boldsymbol{\nabla} \times \boldsymbol{A})+4 \pi \boldsymbol{j}=0 \\
\boldsymbol{\nabla} \cdot \boldsymbol{E}=4 \pi \rho .
\end{array}
$$

The Helmholtz theorem says that a vector field $\boldsymbol{u}$ that vanishes at infinity can be expanded into a sum of its solenoidal $\boldsymbol{u}_{\mathrm{r}}$ and irrotational $\boldsymbol{u}_{\mathrm{g}}$ components. We have for the electric field:

$$
\boldsymbol{E}=\boldsymbol{E}_{\mathrm{r}}+\boldsymbol{E}_{\mathrm{g}},
$$

where

$$
\begin{gathered}
\boldsymbol{\nabla} \cdot \boldsymbol{E}_{\mathrm{r}}=0 \\
\boldsymbol{\nabla} \times \boldsymbol{E}_{\mathrm{g}}=0 .
\end{gathered}
$$

The similar expansion for the vector potential can be written as

$$
\boldsymbol{A}=\boldsymbol{A}_{\mathrm{r}}+\frac{c}{c_{\mathrm{g}}} \boldsymbol{A}_{\mathrm{g}},
$$

where

$$
\begin{gathered}
\boldsymbol{\nabla} \cdot \boldsymbol{A}_{\mathrm{r}}=0 \\
\boldsymbol{\nabla} \times \boldsymbol{A}_{\mathrm{g}}=0 .
\end{gathered}
$$

If we substitute Eqs. (51) and (8) into Eq. (2), we obtain

$$
\frac{1}{c} \frac{\partial \boldsymbol{A}_{\mathrm{r}}}{\partial t}+\boldsymbol{E}_{\mathrm{r}}+\frac{1}{c_{\mathrm{g}}} \frac{\partial \boldsymbol{A}_{\mathrm{g}}}{\partial t}+\boldsymbol{E}_{\mathrm{g}}+\boldsymbol{\nabla} \varphi=0 .
$$

By taking the curl of Eq. (11), we obtain using Eqs. (17) and (10)

$$
\boldsymbol{\nabla} \times\left(\frac{1}{c} \frac{\partial \boldsymbol{A}_{\mathrm{r}}}{\partial t}+\boldsymbol{E}_{\mathrm{r}}\right)=0 .
$$

On the other hand, from Eqs. (6) and (91), we have

$$
\boldsymbol{\nabla} \cdot\left(\frac{1}{c} \frac{\partial \boldsymbol{A}_{\mathrm{r}}}{\partial t}+\boldsymbol{E}_{\mathrm{r}}\right)=0 .
$$


If the divergence and curl of a field are zero everywhere, then that field must vanish. Hence, Eqs. (12) and (13) imply that

$$
\frac{1}{c} \frac{\partial \boldsymbol{A}_{\mathrm{r}}}{\partial t}+\boldsymbol{E}_{\mathrm{r}}=0 .
$$

We subtract Eq. (14) from Eq. (11) and obtain

$$
\frac{1}{c_{\mathrm{g}}} \frac{\partial \boldsymbol{A}_{\mathrm{g}}}{\partial t}+\boldsymbol{E}_{\mathrm{g}}+\nabla \varphi=0 .
$$

Similarly, if we express the current density as

$$
\boldsymbol{j}=\boldsymbol{j}_{\mathrm{r}}+\boldsymbol{j}_{\mathrm{g}}
$$

where

$$
\begin{gathered}
\boldsymbol{\nabla} \cdot \boldsymbol{j}_{\mathrm{r}}=0 \\
\boldsymbol{\nabla} \times \boldsymbol{j}_{\mathrm{g}}=0
\end{gathered}
$$

Eq. (3) can be written as two equations

$$
\begin{gathered}
\frac{\partial \boldsymbol{E}_{\mathrm{r}}}{\partial t}-c \boldsymbol{\nabla} \times\left(\boldsymbol{\nabla} \times \boldsymbol{A}_{\mathrm{r}}\right)+4 \pi \boldsymbol{j}_{\mathrm{r}}=0 \\
\frac{\partial \boldsymbol{E}_{\mathrm{g}}}{\partial t}+4 \pi \boldsymbol{j}_{\mathrm{g}}=0 .
\end{gathered}
$$

From Eqs. (15) and (6), Eq. (44) can be expressed as

$$
\boldsymbol{\nabla} \cdot \boldsymbol{E}_{\mathrm{g}}=4 \pi \rho .
$$

\section{WAVE EQUATIONS FOR THE TWO-SPEED EXTENSION OF ELECTRODYNAMICS}

We will derive from Eqs. (14), (15), (19), (20), and (21) the wave equations for the solenoidal (transverse) and irrotational (longitudinal) components of the fields. In what follows we will use the general vector relation

$$
\boldsymbol{\nabla}(\boldsymbol{\nabla} \cdot \boldsymbol{u})=\nabla^{2} \boldsymbol{u}+\boldsymbol{\nabla} \times(\boldsymbol{\nabla} \times \boldsymbol{u})
$$

The wave equation for $\boldsymbol{A}_{\mathrm{r}}$ can now be found. We differentiate Eq. (14) with respect to time:

$$
\frac{1}{c} \frac{\partial^{2} \boldsymbol{A}_{\mathrm{r}}}{\partial t^{2}}+\frac{\partial \boldsymbol{E}_{\mathrm{r}}}{\partial t}=0 .
$$


We next substitute Eq. (19) into Eq. (23) and use Eqs. (22) and (9) to obtain

$$
\frac{\partial^{2} \boldsymbol{A}_{\mathrm{r}}}{\partial t^{2}}-c^{2} \nabla^{2} \boldsymbol{A}_{\mathrm{r}}=4 \pi c \boldsymbol{j}_{\mathrm{r}}
$$

The wave equation for $\boldsymbol{E}_{\mathrm{r}}$ can be found as follows. We differentiate Eq. (19) with respect to time:

$$
\frac{\partial^{2} \boldsymbol{E}_{\mathrm{r}}}{\partial t^{2}}-c \boldsymbol{\nabla} \times\left(\boldsymbol{\nabla} \times \frac{\partial \boldsymbol{A}_{\mathrm{r}}}{\partial t}\right)+4 \pi \frac{\partial \boldsymbol{j}_{\mathrm{r}}}{\partial t}=0
$$

and substitute Eq. (14) into Eq. (25). By using Eqs. (22) and (6), we obtain

$$
\frac{\partial^{2} \boldsymbol{E}_{\mathrm{r}}}{\partial t^{2}}-c^{2} \boldsymbol{\nabla}^{2} \boldsymbol{E}_{\mathrm{r}}=-4 \pi \frac{\partial \boldsymbol{j}_{\mathrm{r}}}{\partial t} .
$$

In the absence of the electric current, Eqs. (24) and (26) are wave equations for the solenoidal fields $\boldsymbol{A}_{\mathrm{r}}$ and $\boldsymbol{E}_{\mathrm{r}}$.

To find wave equations for the irrotational fields, we need a gauge relation. Substituting (8) into Eq. (11) we get the longitudinal gauge

$$
\boldsymbol{\nabla} \cdot \boldsymbol{A}_{\mathrm{g}}+\frac{1}{c_{\mathrm{g}}} \frac{\partial \varphi}{\partial t}=0 .
$$

The solenoidal part of the vector potential automatically satisfies the Coulomb gauge, Eq. (91). The wave equation for $\boldsymbol{A}_{\mathrm{g}}$ can be found as follows. We first differentiate Eq. (15) with respect to time:

$$
\frac{1}{c_{\mathrm{g}}} \frac{\partial^{2} \boldsymbol{A}_{\mathrm{g}}}{\partial t^{2}}+\frac{\partial \boldsymbol{E}_{\mathrm{g}}}{\partial t}+\frac{\partial \boldsymbol{\nabla} \varphi}{\partial t}=0 .
$$

We then take the gradient of Eq. (27),

$$
\boldsymbol{\nabla}\left(\boldsymbol{\nabla} \cdot \boldsymbol{A}_{\mathrm{g}}\right)+\frac{1}{c_{\mathrm{g}}} \nabla \frac{\partial \varphi}{\partial t}=0,
$$

and combine Eqs. (28), (29) and (20). If we use Eqs. (22) and (10), we obtain

$$
\frac{\partial^{2} \boldsymbol{A}_{\mathrm{g}}}{\partial t^{2}}-c_{\mathrm{g}}^{2} \nabla^{2} \boldsymbol{A}_{\mathrm{g}}=4 \pi c_{\mathrm{g}} \boldsymbol{j}_{\mathrm{g}}
$$

Next, we will find the wave equation for $\varphi$. We take the divergence of Eq. (15),

$$
\frac{1}{c_{\mathrm{g}}} \frac{\partial \boldsymbol{\nabla} \cdot \boldsymbol{A}_{\mathrm{g}}}{\partial t}+\boldsymbol{\nabla} \cdot \boldsymbol{E}_{\mathrm{g}}+\nabla^{2} \varphi=0
$$

and combine Eqs. (31), (27), and (21):

$$
\frac{\partial^{2} \varphi}{\partial t^{2}}-c_{\mathrm{g}}^{2} \nabla^{2} \varphi=4 \pi c_{\mathrm{g}}^{2} \rho
$$


Equations (32) and (30) give wave equations for $\varphi$ and $\boldsymbol{A}_{\mathrm{g}}$.

We may try to find a wave equation for $\boldsymbol{E}_{\mathrm{g}}$ using Eq. (15) in Eqs. (32) and (301). However, in the absence of the charge, we have from Eq. (21)

$$
\boldsymbol{\nabla} \cdot \boldsymbol{E}_{\mathrm{g}}=0 .
$$

Hence, by Eqs. (33) and (7), we have

$$
\boldsymbol{E}_{\mathrm{g}}=0 \text {. }
$$

We see that Maxwell's equations (22)-(44) with the longitudinal gauge (27) imply that the solenoidal and irrotational components of the fields propagate with different velocities. The solenoidal components $\boldsymbol{A}_{\mathrm{r}}$ and $\boldsymbol{E}_{\mathrm{r}}$ propagate with the speed $c$ of light, and the irrotational component $\boldsymbol{A}_{\mathrm{g}}$ of the magnetic vector potential and the scalar potential $\varphi$ propagate with the speed $c_{\mathrm{g}}$.

\section{SINGLE-PARAMETER ELECTRODYNAMICS}

In reality, electrodynamics has only one parameter, the speed of light, $c$. Then, to construct from the above the classical theory, we have to choose among two variants: two waves with equal speeds or a single wave. If we let

$$
c_{\mathrm{g}}=c,
$$

the two-parameter form (11) becomes the familiar Lorenz gauge

$$
\boldsymbol{\nabla} \cdot \boldsymbol{A}+\frac{1}{c} \frac{\partial \varphi}{\partial t}=0 .
$$

Another possible choice is

$$
c_{\mathrm{g}} \gg c \text {. }
$$

The condition (37) turns Eq. (II) into the Coulomb gauge

$$
\nabla \cdot \boldsymbol{A}=0
$$

Substituting

$$
c_{\mathrm{g}}=\infty
$$


into the dynamic equation (32), we get

$$
-\nabla^{2} \varphi=4 \pi \rho
$$

Validity of Eq. (40) for the case when $\varphi$ and $\rho$ may be functions of time $t$ means that the scalar potential $\varphi$ acts instantaneously.

Substituting (39) into Eq. (27) we get for the irrotational part of the vector potential:

$$
\nabla \cdot \boldsymbol{A}_{\mathrm{g}}=0
$$

Insofar as the divergence (41) and the curl (10) of $\boldsymbol{A}_{\mathrm{g}}$ are vanishing, we have

$$
\boldsymbol{A}_{\mathrm{g}}=0
$$

So, on the first sight by (39) irrotational component $\boldsymbol{A}_{\mathrm{g}}$ of the vector potential propagates instantaneously. However, according to relation (42), with (39) $\boldsymbol{A}_{\mathrm{g}}$ vanishes.

Putting (42) into (8) we get

$$
\boldsymbol{A}=\boldsymbol{A}_{\mathrm{r}}
$$

Substituting (43) into Eq. (24) gives

$$
\frac{\partial^{2} \boldsymbol{A}}{\partial t^{2}}-c^{2} \nabla^{2} \boldsymbol{A}=4 \pi c \boldsymbol{j}_{\mathrm{r}}
$$

Eq. (44) indicates that in the Coulomb gauge (38) the vector potential $\boldsymbol{A}$ propagates at speed $c$.

\section{MECHANICAL INTERPRETATION}

Recently, we have shown [6] that in the Coulomb gauge electrodynamics is isomorphic to the elastic medium that is stiff to compression yet liable to shear deformations. In this analogy the vector potential corresponds to the velocity and the scalar potential to the pressure of the medium. Clearly, in an incompressible medium there is no longitudinal waves, the pressure acts instantaneously, and the transverse wave spreads at finite velocity. This mechanical picture provides an intuitive support to the electrodynamic relations (38), (40) and (44) just obtained. 


\section{CONCLUSION}

By using a two-parameter Lorenz-like gauge, we extended electrodynamics to a two-speed theory. Turning the longitudinal speed parameter to infinity we come to electrodynamics in the Coulomb gauge. In this way we show that the scalar potential acts instantaneously while the vector potential propagates at speed of light.

\section{Acknowledgments}

I would like to express my gratitude to Dr. I. P. Makarchenko for valuable comments concerning the non-existence of longitudinal waves of the electric field and longitudinal waves in the Coulomb gauge.

\section{References}

[1] Jackson J D "From Lorenz to Coulomb and other explicit gauge transformations" 2002 Am. J. Phys. $\mathbf{7 0} 917$

[2] Stewart A M "Vector potential of the Coulomb gauge" 2003 Eur. J. Phys. 24519

[3] Drury David M "Irrotational and solenoidal components of Maxwell's equations" 2002 Galilean Electrodynamics 1372

[4] Chubykalo Andrew E and Onoochin Vladimir V "On the theoretical possibility of the electromagnetic scalar potential wave spreading with an arbitrary velocity in vacuum," 2002 Hadronic Journal, 25597 Preprint physics/0204062

[5] Jackson J D "Criticism of "Necessity of simultaneous co-existence of instantaneous and retarded interactions in classical electrodynamics" by Andrew E. Chubykalo and Stoyan J. Vlaev" 2002 Int.J.Mod.Phys. A17 3975 Preprint hep-ph/0203076

[6] Dmitriyev Valery P "Electrodynamics and elasticity" 2003 Am.J.Phys. 71952 